\title{
2006-1674: SHARING SOFTWARE ENGINEERING CURRICULUM MATERIALS
}

\section{Gregory Hislop, Drexel University}

Greg Hislop is on the faculty of the College of Information Science and Technology at Drexel University. He leads the college efforts in a BS and MS in software engineering offered jointly with the Department of Computer Science.

\section{Mark Sebern, Milwaukee School of Engineering} Mark Sebern is on the faculty of the Department of Electrical Engineering and Computer Science at the Milwaukee School of Engineering. He directs the BS in Software Engineering.

\section{Michael Lutz, Rochester Institute of Technology}

Mike Lutz is on the faculty of the Golisano College of Computer and Information Sciences at Rochester Institute of Technology. He led development of the first BS in Software Engineering in the United States. 


\section{Sharing Software Engineering Curriculum Materials}

\section{Introduction}

In addition to the usual economies of scale, sharing curriculum materials is particularly important for software engineering since the pool of faculty is rather small and software engineering curriculum materials age quickly. This means that broad engagement by faculty with the question of how to promote material sharing is essential for the growth of software engineering education. This paper is intended to foster discussion within the software engineering community about developing and maintaining shared curriculum resources on an on-going basis.

The paper approaches this topic by summarizing the experience of the SWENET project in creating shared curriculum materials for software engineering. SWENET, The Network Community for Software Engineering Education, was an NSF funded project to develop curriculum modules for faculty members wanting to incorporate software engineering concepts in new or existing courses. The paper discusses the project results, focusing on lessons learned.

Although the benefit of sharing course materials is obvious, the practice is not particularly wide spread in higher education. Reasons for this low level of sharing are discussed in terms of faculty motivators and inhibitors. The online environment developed by the project for course materials collection will also be discussed along with an examination of the strengths and weaknesses of the approach taken. In addition, the project employed various mechanisms to encourage faculty participation, including hosting several summer workshops for faculty. The effectiveness of these mechanisms will be examined. The paper also includes suggestions and ideas for future efforts to share curricular materials.

\section{Project Summary}

SWENET, the Network Community for Software Engineering Education, was an NSF funded project to produce and organize high-quality materials supporting software engineering education. The project sought to support faculty members delivering software engineering degrees, however, the project also maintained a focus on accommodating faculty who teach in other computing degree programs where the need for software engineering coverage is high, but the available class time for these topics is much lower. SWENET was a multi-institution effort, encompassing several of the first B.S. in Software Engineering programs in the United States.

The SWENET effort to create a collection of curriculum materials included creation of a Web site (http://www.swenet.org) and structure for defining and organizing the materials. The materials were organized into course modules with a fixed structure anchored to an existing framework. The project began shortly after the Guide to the Software Engineering Body of Knowledge project (SWEBOK) ${ }^{1}$ was released, so SWEBOK provided the initial framework for the project. The module categories in the prototype web site - design, process, quality, and requirements - corresponded directly to major focus areas of SWEBOK. 
More recently, the Computing Curriculum in Software Engineering (SE 2004) ${ }^{2}$ became available and influenced the development of the SWENET project. SE 2004 gave rise to a more detailed body of knowledge for education. This software engineering education body of knowledge (or SEEK) had the advantages of (a) relating directly to the mission of SWENET, and (b) providing a more structured and focused taxonomy. SWENET was adjusted to use the SEEK areas and topics to structure the modules and to help focus the module development effort.

The SWENET project was publicized via various presentations and workshops held at conferences and other meetings including Frontiers in Education ${ }^{3}$ and the Conference on Software Engineering Education and Training ${ }^{4}$.

SWENET also sponsored several multi-day workshops to promote interaction among faculty with software engineering education interests. In 2004, a workshop was held at Rochester Institute of Technology focusing on approaches to adding software engineering content to degree programs. This workshop addressed a range of options including complete software engineering degrees, minors in software engineering, and adding coverage of software engineering in other degree programs such as computer science.

In summer of 2005, a workshop was held at Milwaukee School of Engineering to allow faculty to work together on curriculum materials development, and to discuss issues in teaching various topics in software engineering. In addition to learning the SWENET approach to organizing and describing curriculum materials, participants spent time creating new materials and packaging existing materials.

Both the curriculum development efforts and the workshops have attracted substantial interest among software engineering educators. .

\section{Lessons Learned}

The SWENET project produced a variety of good results. Even so, the effort to create a community of software engineering educators is just beginning. It is important to critique the SWENET effort as part of determining how to continue efforts of this type. This section identifies and discusses various aspects of the SWENET project that worked well or not as well as expected.

Infrastructure - Substantial effort was made to define a curriculum module structure and to represent that structure on the Web site. The intention was to support organization and retrieval of course materials, and to ensure that modules would be defined in a way that would help to make them usable. It appears that the project was successful in defining a useful structure for the project goals. However, in retrospect it appears that the infrastructure development approach had some disadvantages:

- Development Effort - The effort consumed a lot of the available project time and resources, particularly in light of the number of modules that have been contributed thus far. If the repository continues to grow and be used, the investment in infrastructure will 
have been well worth it. At present however, the investment is hard to justify by the amount of content or level of use.

- Involvement - During the development effort, it was difficult to involve people beyond the SWENET team in meaningful ways. This delayed and limited participation of the wider community of software engineering educators.

- Ease of Submission - While the module definition structure helps insure usability, it also creates an additional burden for someone submitting materials. This burden is not huge, but, particularly for a first submission to the repository, it presents a learning curve for the submitter. This may keep people from contributing to the effort.

- Topical framework - Adopting a topical framework (SEEK) for organizing the materials provided an easily recognizable set of categories. However, this approach does not easily accommodate cross-cutting topics. For example, contributions that might test knowledge of incoming students, or that relate to capstone design courses do not have an obvious category in a software engineering topical structure.

Wiki - Near the end of the project, the fixed structure of module submission was supplemented by a Wiki. The intention was to provide a flexible forum for discussion and contribution of less complete materials as a supplement to the module repository. At the time of this evaluation it is too early to know if this addition is useful.

Modules - One key decision in organizing the materials was to deal with fairly small chunks of material. These "course modules" were intended to contain anything from a single class activity (e.g. an exercise) to about a week of course material. This level of granularity has proved to be very workable, and is well received by the software engineering community. It also avoided the problem of seeming to impose a particular curriculum model as part of the project.

Modules are defined using a standard template that categorizes and describes the module contents. The categorizing fields include a title, version, author, and SEEK areas. The description includes an abstract, learning objectives, topics, a list of the module materials, and references to additional resources. Examples of modules can be found by browsing the SWENET site.

Contribution Level - Throughout the project, the level of contribution of course modules by instructors has been lower than hoped. There are many possible reasons for this including:

- Lack of interest - It is possible that instructors simply aren't interested in having shared course materials. However, presentations at conferences and other gatherings have always seemed to attract substantial interest in sharing course materials

- Small community - The community of software engineering educators is not very large. It may be that it is difficult for a community of this size to create and support an extensive repository of course materials 
- Rewards - For faculty at research universities, teaching activities tend to be rewarded only at very limited levels, and in general this work may be less respected than traditional research. For faculty at teaching institutions, typically higher teaching loads can restrict time available to contribute shared materials.

- Confidence - Some faculty are reluctant to post course materials they have developed due to concern that the materials might not be well received by colleagues.

Dual audience - SWENET was intended to address the needs of instructors in software engineering degrees as well as instructors teaching software engineering in other degree programs. This is an admirable goal, and greatly extends the potential size of the community. On the other hand, the needs of these two groups may be different enough that it is difficult to have a small collection of course materials cover both groups.

Workshops - The SWENET 2004 and 2005 workshops were very effective for advancing the project and for starting to build a community of software engineering educators. The 2005 workshop was quite successful at providing a venue for small teams of faculty members to work on shared teaching interests. The teams were also quite successful at producing course modules for the SWENET site. In retrospect, it would have been effective to hold additional workshops and to hold them earlier in the project.

Visibility - It appears that SWENET became well-known among the core group of software engineering educators who are active at conferences like FIE and CSEET. However, the project seems, even now, to be much less known in the broader audience of computing educators.

\section{Conclusions}

NSF funding for SWENET ended in late 2005, although the Web site is being continued on a volunteer basis. Some possibilities to create forward direction building on the SWENET experience are:

SE 2004 implementation - If the SE 2004 curriculum model is generally accepted by the institutions offering software engineering degrees, it would be natural to build on SWENET to move toward having a full set of sample materials that would support implementation of the model in a BSSE degree program. This approach might prompt much more active involvement by schools offering software engineering degrees.

Case study development - One of the hardest aspects of curriculum development is finding good data and examples for course use. The course module approach taken in SWENET produced modules that contain both data and examples. But there is still a pressing need for more extensive case studies that can be used to provide students with a better understanding of the full software life cycle. It is particularly important that materials that work with a realistic size system be developed.

Permanent home - Maintaining SWENET as a volunteer effort is probably a weak model for the long term. Planning underway to have the site taken over by one of the computing 
professional societies is essential to maintaining the project and making it more visible. This connection will also help provide career value to participation efforts by volunteers.

Scholarship of teaching - Projects like SWENET fit nicely with the movement toward a scholarship of teaching ${ }^{5}$. In this model, a key aspect of evaluating teaching scholarship is that the work is made public and reviewed by peers. The software engineering education community could foster this scholarly review by actively sharing and reviewing curricular materials.

Faculty workshops - The strong interest in the various SWENET workshops makes the option of holding additional workshops seem attractive. Funding for additional workshops is likely to be limited at best, so a model for self-supporting workshops is needed.

\section{References}

1. Bourque, Pierre, and Dupuis, Robert (eds.), "Guide to the Software Engineering Body of Knowledge, 2004 Version," IEEE CS Press 2004.

2. Diaz-Herrera, Jorge L., and Hilburn, Thomas B., "Computing Curricula: Software Engineering" http://sites.computer.org/ccse.

3. Lutz, Michael J., Thomas B. Hilburn, Gregory W. Hislop, W. Michael McCracken, Mark J. Sebern. “The SWENET Project: Bridging the Gap from Bodies of Knowledge to Curriculum Development."

Proceedings, 33rd ASEE/IEEE Frontiers in Education. IEEE CS Press. November 2003.

4. Hislop, Gregory W., Thomas B. Hilburn, Michael J. Lutz, W. Michael McCracken, Mark J. Sebern. "Software Engineering Course Module Development Workshop." Proceedings, 17th Conference on Software Engineering Education and Training. IEEE CS Press. March 2004.

5. Boyer, Ernest L. "Scholarship Reconsidered: Priorities of the Professoriate" Princeton: The Carnegie Foundation for the Advancement of Teaching. 1990. 